\title{
Self-assembling unsymmetrical bis-ureas
}

Article

Accepted Version

Creative Commons: Attribution-Noncommercial-No Derivative Works 4.0

Melia, K., Greenland, B. W., Hermida-Merino, D., Hart, L. R., Hamley, I. W., Colquhoun, H. M., Slark, A. T. and Hayes, W. (2018) Self-assembling unsymmetrical bis-ureas. Reactive and functional polymers, 124 (1). pp. 156-161. ISSN 1381-5148 doi: https://doi.org/10.1016/j.reactfunctpolym.2018.01.017 Available at https://centaur.reading.ac.uk/75314/

It is advisable to refer to the publisher's version if you intend to cite from the work. See Guidance on citing.

To link to this article DOI: http://dx.doi.org/10.1016/j.reactfunctpolym.2018.01.017

Publisher: Elsevier

All outputs in CentAUR are protected by Intellectual Property Rights law, including copyright law. Copyright and IPR is retained by the creators or other copyright holders. Terms and conditions for use of this material are defined in the End User Agreement.

\section{www.reading.ac.uk/centaur}

\section{CentAUR}

Central Archive at the University of Reading

Reading's research outputs online 


\title{
Self-Assembling Unsymmetrical Bis-Ureas
}

Kelly Melia, ${ }^{a}$ Barnaby W. Greenland, ${ }^{\mathrm{b}}$ Daniel Hermida-Merino, ${ }^{\mathrm{c}}$ Lewis R. Hart, ${ }^{\mathrm{a}}$ Ian W. Hamley, ${ }^{\mathrm{a}}$ Howard M. Colquhoun, ${ }^{\mathrm{a}}$ Andrew T. Slark, ${ }^{\mathrm{d}}$ and Wayne Hayes ${ }^{\mathrm{a} *}$

\footnotetext{
a. Department of Chemistry, University of Reading, Whiteknights, Reading, RG6 6AD, U.K.

b The Reading School of Pharmacy, University of Reading, Whiteknights, Reading, RG6 6AD, U.K

${ }^{c}$ ESRF, 6 Rue Jules Horowitz, BP 220, F-38043 Grenoble Cedex 09, France.

d. Henkel UK Ltd, 957 Buckingham Avenue, Slough, SL1 4NL, Berks, U.K.

* Fax: +44 118378 6331; Email: w.c.hayes@ reading.ac.uk
}

\begin{abstract}
Model compounds of unsymmetrical bis-ureas have been designed and synthesised to investigate and further understand the formation of supramolecular polymer arrays. Association constants $\left(<200 \mathrm{M}^{-1}\right)$ for the bis-ureas have been determined by ${ }^{1} \mathrm{H}$ NMR spectroscopy, and further characterised by optical spectroscopy. In addition, SAXS analysis of the bulk material reveals the formation of different highly ordered structures demonstrating complex hydrogen bonding of these unsymmetrical materials. Thermal transitions are also observed in some examples of unsymmetrical bis-ureas near to the melting point, as evidenced by double endotherms in DSC analysis. We also propose a re-organisation mechanism whereby the supramolecular array is first altered before proceeding to melt which is supported by variable temperature SAXS analysis.
\end{abstract}

Key Words: Unsymmetrical, Bis-Urea, Hydrogen Bonding, Self-Assembly, Association Constant.

\section{Introduction}

Supramolecular chemistry has found widespread use in applications ranging from drug delivery systems, composite materials, and sensors.[1-4] Reversible, non-covalent interactions such as hydrogen bonding[5] and $\pi-\pi$ stacking interactions[6] are able to be tuned to afford arrays which are responsive and reversible.[7-9] Moreover, multiple hydrogen bonds between low molecular weight dimers result in increased strength in the supramolecule,[10] where the association constants $\left(K_{a}\right)$ can reach as high as $>10^{6} \mathrm{M}^{-1}$ as reported by Meijer[11] and $10^{7} \mathrm{M}^{-1}$ as reported by Zimmerman.[12]

Several studies focused on the assembly characteristics of low molecular weight molecules that feature either urethane or urea functionalities have been developed.[13-15] In particular, urea analogues[1619] which possess accepting bifurcated geometries demonstrate efficient self-association and have greater potential for hydrogen bonding than the analogous urethane moiety. Hydrogen bonding motifs have been used extensively to create stable networks [20-22] which, even at low concentrations, provide evidence of assembly into higher ordered structures via self-assembly of the urea 
moieties.[23] These self-assembled arrays have found use in a wide range of applications including materials for water purification[24] and molecular switches.[23]

Hydrogen bonded arrays that incorporate multiple urea groups have also been reported.[20,25] Bouteiller and co-workers have demonstrated[15] that for bis-urea systems, including a conformationally controlled architecture, control over the macroscopic structure is possible by careful design of the substituents in the ortho-positions on the aryl moiety; thus imparting some level of preorganisation in the small molecules, resulting in hydrogen bond array formation. Similarly, Meijer, Sijbesma and co-workers have also reported[26,27] bis-urea derived thermoplastic elastomers which are capable of self-sorting by virtue of the spacer between the urea functionalities.

However, bis-ureas described in the literature focus on symmetrical structures,[28,29] and these can lead to the enhancement of high melt viscosity with processing disadvantages. Herein we report the design, synthesis and self-assembly studies of a series of structurally related unsymmetrical bis-ureas as a convenient way of incorporating multiple hydrogen bonds into a molecule whilst controlling the molecular architecture and maintaining a straightforward synthetic procedure.

\section{Experimental}

All reagents and solvents were purchased from Acros Organics or Sigma Aldrich and used as received, with the exception of Tetrahydrofuran (THF) which was distilled from sodium and benzophenone prior to use. General characterisation by TLC was conducted using TLC silica gel 60 F254 aluminium backed sheets purchased from Fisher Scientific and visualised using an ultraviolet light source (254 $\mathrm{nm})$ and/or potassium permanganate staining. Preparative TLC was undertaken using silica gel GF 20 $\times 20 \mathrm{~cm} 1000 \mu \mathrm{m}$ plates backed on glass. Purification by column chromatography was carried out using silica gel 60. ${ }^{1} \mathrm{H}$ NMR spectroscopy (400 MHz) and ${ }^{13} \mathrm{C}$ NMR spectroscopy (100 MHz) were recorded with either a Bruker Nanobay 400, or Bruker DPX 400, using an appropriate deuterated solvent. Infrared spectra were collected using a Perkin-Elmer Spectrum 100 FTIR spectrometer with an ATR attachment. Mass spectrometric (MS) analysis was conducted using a ThermoFisher Scientific Orbitrap XL mass spectrometer operating in electrospray ionisation (ESI) mode. Parent mass ions, dimers or principal fragments are quoted. Association constants $\left(\mathrm{K}_{\mathrm{a}}\right)$ were determined using ${ }^{1} \mathrm{H}$ NMR spectroscopy, using known sample concentrations in deuterated chloroform. The change in chemical shift of up to three proton resonances over the observed concentration range (a minimum of 10 data points between $0.5 \mathrm{mg} / \mathrm{mL}$ up to $80 \mathrm{mg} / \mathrm{mL}$, depending on solubility) were input into the Dynafit[30] software suite in order to generate an association constant with units of $\mathrm{M}^{-1}$. Differential Scanning Calorimetry (DSC) thermograms were obtained using a TA DSC Q2000 adapted with a TA Refrigerated Cooling System 90, using aluminium TA Tzero pans and lids. Thermal transitions were recorded using heating and cooling rates of $5{ }^{\circ} \mathrm{C} \mathrm{min}^{-1}$. Small-Angle X-ray Scattering and Wide-Angle 
X-ray Scattering (SAXS/WAXS) experiments were performed on beamline BM26B at ESRF, Grenoble, France. Samples, as powders, were placed in aluminium DSC pans with mica windows and mounted in a brass block for temperature control. The sample-to-detector (SAXS) distance was $2 \mathrm{~m}$, the sample-to-detector (WAXS) distance was $26 \mathrm{~cm}$ and the X-ray energy was $12 \mathrm{keV}$. The $\mathrm{q}=4 \pi \sin$ $\theta / \lambda$ range was calibrated using silver behenate. Data processing (background subtraction, radial averaging) was performed using SAXSUtilities software. Computational modelling (molecular mechanics with charge-equilibration) was carried out using the "Cerius2" suite of programmes from Accelrys Inc. (now Biovia), San Diego, running on a Silicon Graphics O2 system. The force field used was "Dreiding II".[31] Simulations involved the creation of an ensemble of oligomers that were then positioned at approximately van der Waals distances from one another. Energy-minimisation of the ensemble (both intra- and inter-molecular) was then carried out using the "smart minimiser" algorithm, without constraints, to high convergence.

\section{General method for the synthesis of mono hydroxyl-alkyl MDI ureas:}

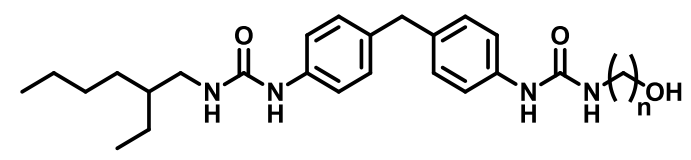

Figure 1: General structure of 4,4'-MDI derived bis-ureas showing the solubilising ethylhexyl end-group and linear hydroxyalkyl chains range from $\mathrm{n}=2$ to $n=5$.

To a stirred solution of 4,4'-methylenebis(phenyl isocyanate) 1 (1 equiv.) in dry THF under nitrogen was added drop wise 2-ethyl-1-hexylamine 3 (1.05 equiv.) and hydroxyalkyl amine 4-7 (1 equiv.) dissolved in dry THF. The reaction was heated under reflux until the isocyanate absorbance band $\left(\sim 2250 \mathrm{~cm}^{-1}\right)$ was not evident in the IR spectrum, (between 15 minutes -5 hours). The reaction mixture was then cooled to room temperature and the precipitate thus formed removed by filtration. The filtrate was dried under reduced pressure and then washed in chloroform, filtered and dried under reduced pressure to yield the desired compound (See Figure 1 and S1-8).

\section{General method for synthesis of mono hydroxyl-alkyl HDI ureas:}

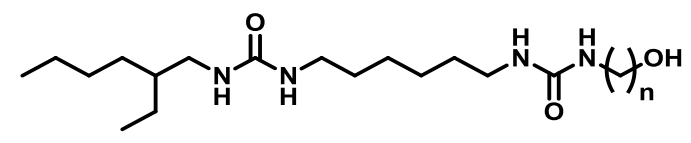

Figure 2: General structure of HDI derived bis-ureas showing the solubilising ethylhexyl end-group and linear hydroxyalkyl chains range from $\mathrm{n}=2$ to $n=5$.

To a stirred solution of hexamethylene diisocyanate 2 ( 1 equiv.) in dry THF under nitrogen was added drop wise hydroxyalkyl amine 4-7 (1 equiv.) and 2-ethyl-1-hexylamine 3 (1.05 equiv.) dissolved in dry THF. The reaction was heated at a temperature of $60{ }^{\circ} \mathrm{C}$ until the isocyanate peak $\left(\sim 2250 \mathrm{~cm}^{-1}\right)$ 
was no longer observed in the IR spectrum, (between 15 minutes - 5 hours). The reaction mixture was then cooled to room temperature and a series of washes (Figure 2 and S9-16) performed to give the desired compound. Full synthetic procedures and characterization for the unsymmetrical bis-ureas and model compounds can be found in the Supporting Information.

\section{Results and Discussion}

Previous studies have shown that bis-urea type compounds, synthesised from diisocyanates and primary amines, provide access to enhanced hydrogen bonding interactions.[29,32,33] This well documented synthetic protocol has found extensive use within the adhesive industry, amongst others, and has been exploited using commercially available precursors. However, unsymmetrical bis-ureas are rarely reported despite the increased variety of structures which can be achieved by asymmetrical substitution of a diisocyanate. In order to create a facile method for synthesising un-symmetrical ureas, the design of the molecule and end groups is paramount. Diisocyanates offer a facile route to synthesising bis-ureas, and indeed this route is often employed in industrial settings to great effect. An aromatic (4,4'-methylenebis(phenyl isocyanate) [4,4'-MDI]) $\mathbf{1}$ and an aliphatic (1,6-hexamethylene diisocyanate [HDI]) 2 (Scheme 1) were selected to assess the contribution of $\pi-\pi$ stacking interactions toward the overall properties of the self-assembling array afforded. To improve the solubility of the bis-urea, which can be problematic owing to highly direction and specific interactions yielding insoluble arrays, a branched alkyl group, namely an ethyl hexyl motif $\mathbf{3}$ was selected as one terminus for the small molecule. Finally, a second end-group containing an alkyl chain and reactive handle, namely alcohols 4-7 (Scheme 1) for further subsequent chemistries to take place was required to allow for their use in value added applications. The aforementioned considerations were then used to generate the generic structure (Figure 3) which could then be used to examine the properties of the resulting self-assembled bis-urea arrays.

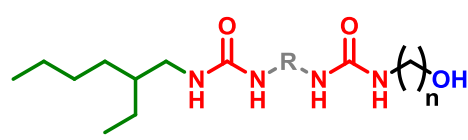

Figure 3: The target structure identified for bis-ureas reported in this study, where R constitutes 4,4'-MDI or HDI moieties and the linear alkyl chains range from $\mathrm{n}=2$ to $n=5$.

Utilising the highly efficient reaction between isocyanates and amines, a library of unsymmetrical bis-ureas (8-15) have been synthesised (Scheme 1) via a simple one pot strategy as reported previously[14,34]. Statistical addition of the two different amine end-groups resulted in a maximum theoretical yield of $50 \%$ of the desired unsymmetrical bis-urea. Owing to the similar polarities and $\mathrm{R}_{\mathrm{f}}$ values between the symmetric and asymmetric ureas, column chromatography was not a viable method for purification. Therefore, the symmetrical by-products were removed by a series of washes, 
relying on the differing solubilities of the symmetrical bis-ureas and thus allowing for the desired product to be isolated from this statistical synthesis in excellent purity (> 95\%) and acceptable yields (up to $56 \%$ )(See Figures S1-16).
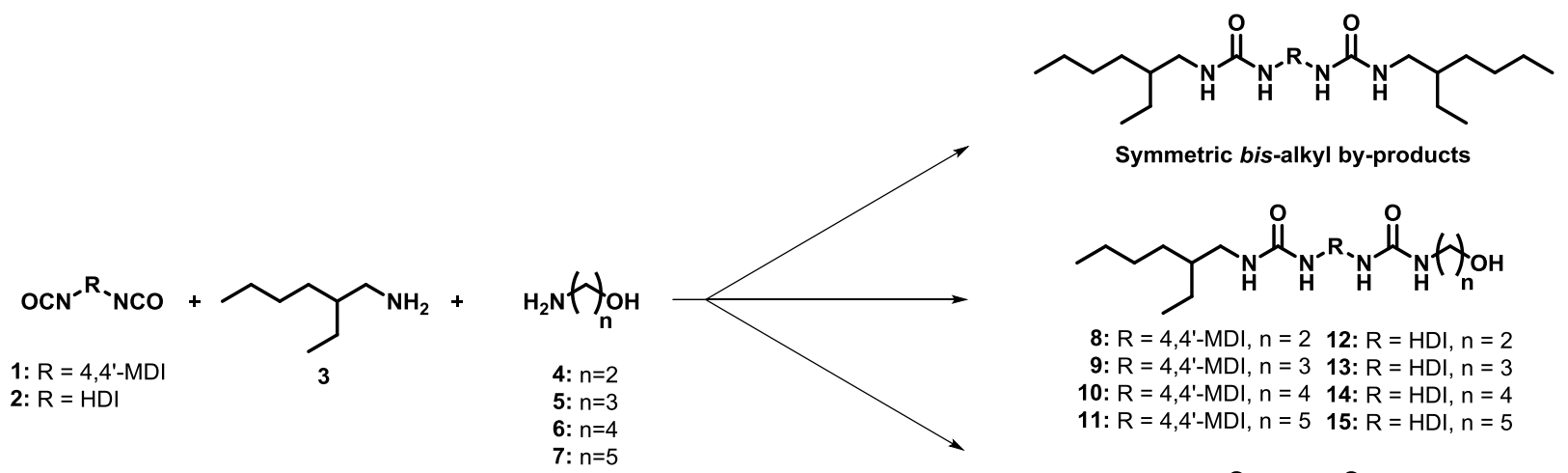

Symmetric bis-alkyl by-products

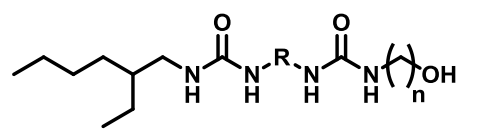

8: $R=4,4^{\prime}-M D I, n=2$ 12: $R=H D I, n=2$

9: $\mathrm{R}=4,4^{\prime}-\mathrm{MDI}, \mathrm{n}=3$ 13: $\mathrm{R}=\mathrm{HDI}, \mathrm{n}=3$

10: $R=4,4-M D I, n=4$ 14: $R=H D I, n=4$

11: $R=4,4^{\prime}-M D I, n=5$ 15: $R=H D I, n=5$

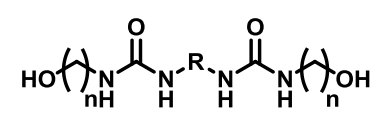

Symmetric bis-hydroxyl by-products

Scheme 1: General reaction scheme for the synthesis of unsymmetrical bis-ureas, assuming equal reactivity of the amines and $100 \%$ consumption of the isocyanate moieties.

Confirmation of the successfully isolated mono hydroxyl-alkyl product bis-ureas (8-15) was first achieved by 2D TLC analysis, which revealed a single spot, demonstrating the removal of the symmetrical by-products. ${ }^{1} \mathrm{H}$ NMR spectroscopy analysis (Figure S1-16) was then used to confirm the desired bis-urea had been obtained. Integration of the terminal methyl protons of the ethyl hexyl endgroups were compared with respect to the protons of the linking moiety (methylene bisphenyl or hexamethylene) adjacent to the urea groups which were in good agreement for the formation of the unsymmetrical bis-urea. This integral ratio was further corroborated by comparison with the methylene protons within the alcohol containing moiety which gave further evidence of successful desymmetrisation. Mass spectrometry was also used to confirm the successful isolation of the desired unsymmetrical bis-urea (See SI). A parent ion for each unsymmetrical bis-urea compound was found in the mass spectra, but crucially mass ions corresponding to the analogous symmetric bis-ureas diols or dialkyl derivatives were not evident, demonstrating that the desired product had indeed been isolated. It was also noted that dimers were present in several of the molecules, indicating the desired high degrees of association in this class of molecules.[35] The relative shift to higher wavenumbers of the broad $\mathrm{OH}$ absorption band at $\sim 3320 \mathrm{~cm}^{-1}$ and the amide II band at $\sim 1570 \mathrm{~cm}^{-1}$ in the mono hydroxy-alkyl bis-urea compounds 8-15 as observed in the IR spectra, provided further evidence that asymmetrical bis-ureas are capable of hydrogen bonding in the bulk.[14]

DSC analysis of the bis-ureas revealed that the compounds appear to reorganise, as evidenced by two endothermic transitions, conceivably into different phase structures or morphologies during the melt, as exemplified in Figure 4 which shows the DSC thermogram for bis-urea 8. Interestingly, this phase 
change phenomena were observed for several of the small molecules synthesised within this study, revealing this structural conversion with respect to temperature is common to bis-ureas. Interestingly, a second melt is observed predominantly in the 1,6-hexamethylene diisocyanate 2 derived bis-ureas, indicating that increased degrees of freedom allows for facile reordering within the supramolecular array at elevated temperatures. A common isomorph transition is also observed in the temperature regime of approximately $116^{\circ} \mathrm{C}\left( \pm 10{ }^{\circ} \mathrm{C}\right.$, resulting from different alkyl chain lengths $)$ demonstrating that the self-assembled arrays share at least one common morphology.

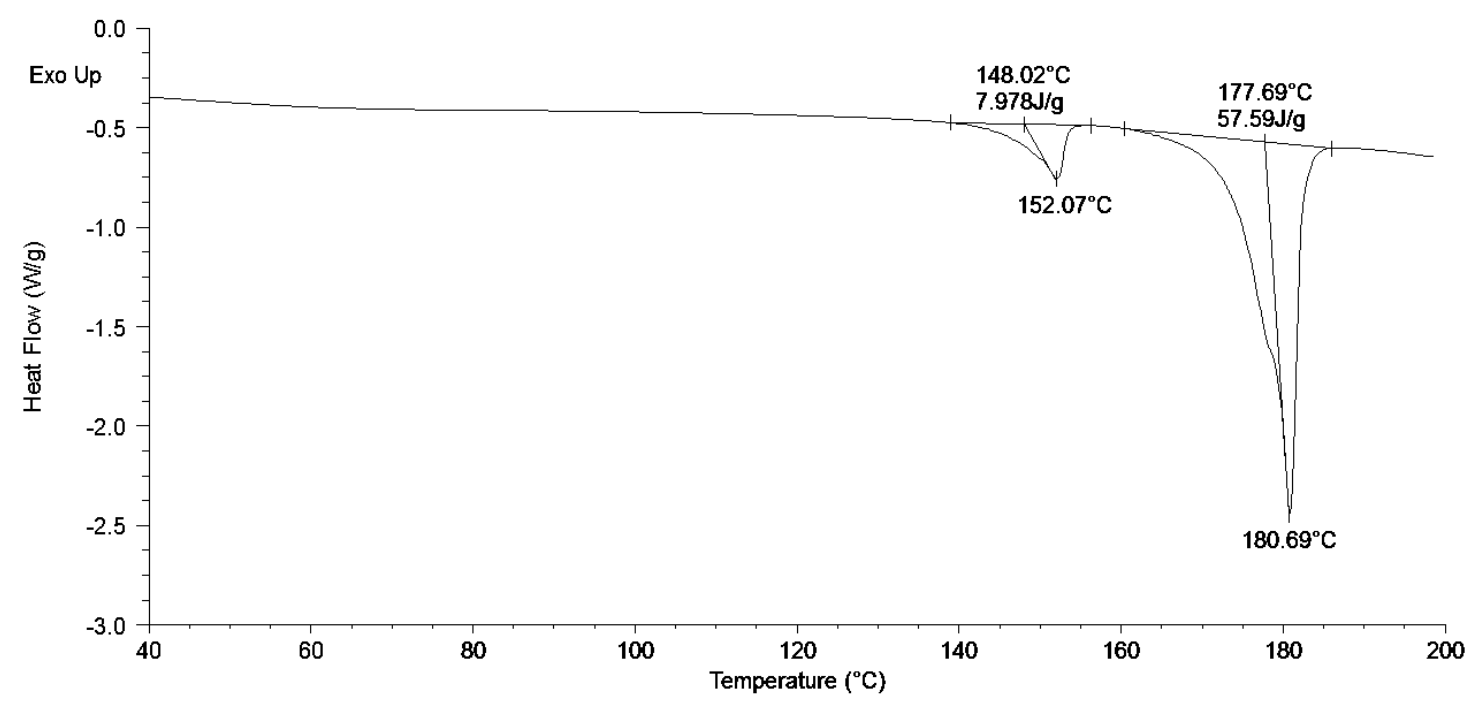

Figure 4: DSC thermogram of compound 8 revealing the double endothermic transition during the melt between $152.1{ }^{\circ} \mathrm{C}$ and $180.7^{\circ} \mathrm{C}$ during the heat cycle, indicative of thermal reorganisation within the supramolecular array formed.

The 4,4'-MDI derived bis-ureas, in general exhibited elevated melting points when compared to the HDI analogues on account of the relatively rigid aromatic units in MDI. A correlation between the length of the alkyl chain and the highest melting point observed by DSC was noted for the unsymmetrical 4,4'-MDI 8-11 and HDI 12-15 derived compounds (Table S1). As the carbon chain between the urea and hydroxyl functionality increases in length, the melting points were seen to decrease as a result of less ordered packing within the supramolecular array. Moreover, this effect was much larger in the 4,4'-MDI derived analogues (Figure 5) where an increase in spacer length from $\mathrm{C}_{2} \mathrm{H}_{4}$ to $\mathrm{C}_{5} \mathrm{H}_{10}$ results in a greater reduction in the upper melting point $\left(-72{ }^{\circ} \mathrm{C}\right)$ when compared to the HDI analogues $\left(-9^{\circ} \mathrm{C}\right)$. The difference between the two series of bis-ureas can be rationalised in terms of the degree of order within the supramolecular arrays. Increasing the chain length of the alcohol bearing side-arm in the 4,4'-MDI derived bis-ureas results in less order in the supramolecular array 
and thus suppresses the melting point. This trend is not as evident in the HDI analogues which have greater degrees of freedom within the structure.

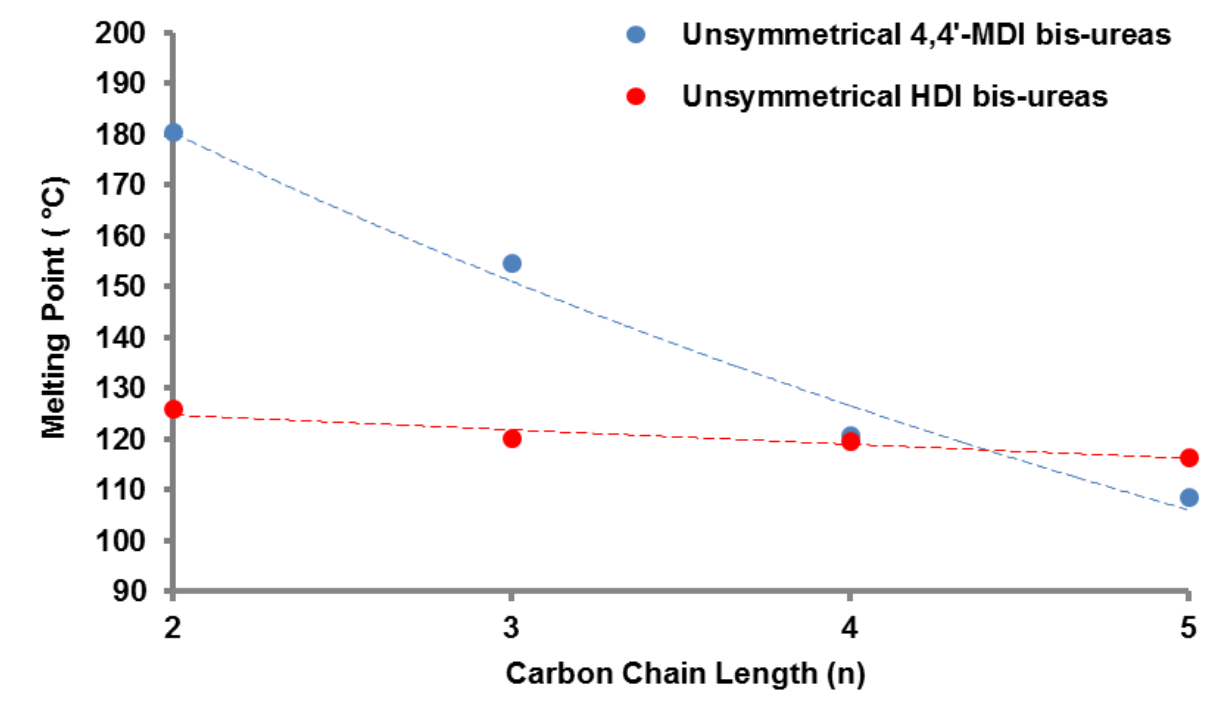

Figure 5: The effect of increasing spacer length on higher melting point for both the 4,4'-MDI and HDI monoalcohol analogues.

The tuneable thermal properties of the unsymmetrical bis-ureas proved to illustrate the advantages of desymmetrisation when compared to the symmetrical analogues, for use in industrial applications such as additives or adhesives. All of the asymmetric bis-ureas have the same general solubility profile and potential for hydrogen bonding, however their melting point, and thus processability, can be varied simply by altering the spacer length, especially for the $4,4^{\prime}$-MDI derivatives.

Molecular models were generated using Cerius ${ }^{2}$ software with the Dreiding II[31] force field to probe the hydrogen bonding interactions of the bis-ureas 8-15 which led to the consideration of complex and varied association phenomena in these molecules comprising unsymmetrical bis-ureas. An ensemble of oligomers that were then positioned at approximately van der Waals distances from one another before energy-minimisation of the ensemble (both intra- and inter-molecular) was then carried out using the "smart minimiser" algorithm, without constraints, to high convergence. This procedure reproducibly yielded the patterns of hydrogen bonds (Figure 6) wherein dimers or oligomers were formed through hydrogen bonding of the urea motifs within the molecules, resulting in aggregation through self-assembly. Using bis-urea $\mathbf{8}$ as a representative example, a bifurcated binding mode was observed between both pairs of ureas in the respective individual molecules, resulting in an end-to-end layered structure being formed (Figure 6a). Molecules were observed to be approximately $7.7 \AA$ apart within the sheet, and the sheet itself being symmetrical with respect to the urea functionalities. Staggered layers were also modelled in which alternate urea end-groups would hydrogen bond with 
one another (Figure 6b). Finally, attention was focused on the urea functionality adjacent to the terminal alcohols. Figure $6 \mathrm{c}$ shows a model of bis-urea $\mathbf{8}$ where not only the ureas, but also the terminal hydroxyl groups are cooperating in the formation of a total of six hydrogen bonds in the dimer. This structure highlights the possibility for highly specific and directional hydrogen bonding interactions arising from these unsymmetrical structures. It is proposed that the actual configuration of these molecules in the bulk is likely to be a combination of different structures, as exemplified by the models and further explored by SAXS analysis.

a

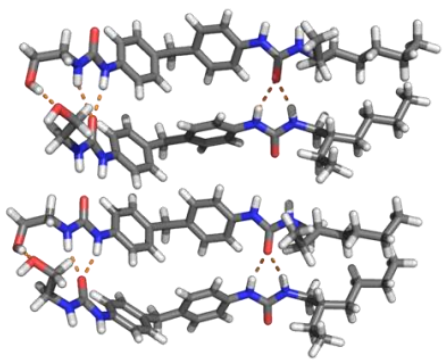

b

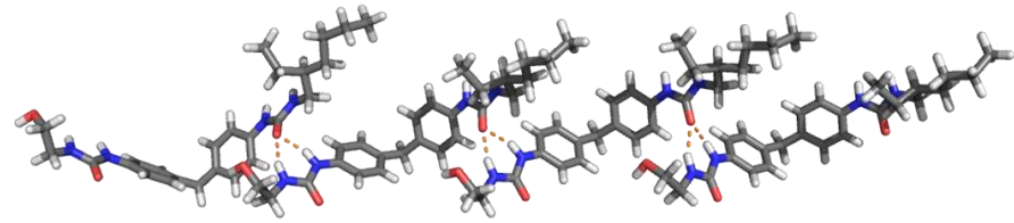

C

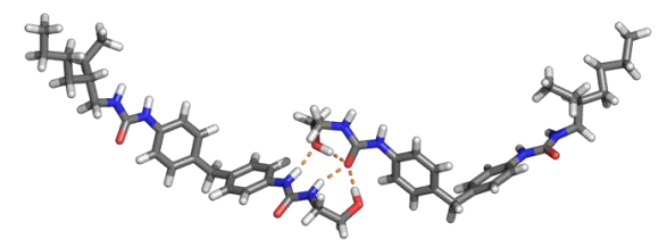

Figure 6: Energy minimised molecular model of compound 8, illustrating a) layered type array with bifurcated hydrogen bonding between ureas b) staggered layered hydrogen bonding structure, where alternate urea functionalities form bifurcated hydrogen bonds, and c) the inverted end-to-end structure where hydrogen bonded dimer formed by cooperation of the terminal hydroxyl groups.

SAXS analysis of the mono hydroxyl-alkyl bis-ureas 8-15 revealed the formation of ordered structures, as predicted by molecular modelling (Table S2). To exemplify this, the SAXS intensity profile of compound 8 (Figure 7a) revealed d-spacings of $50.3 \AA$, $27.9 \AA, 18.3 \AA, 13.7 \AA$, and $11.0 \AA$. These can be indexed to a lamellar structure, considering a small $(6 \%)$ error in the position of the first order peak. The signal at $18.3 \AA$ is likely to represent the molecular length of the molecule when considering the energy minimised conformation (between 18.2-19.5 $\AA$ ). A tetramer in a staggered layer conformation exhibits a length of $52.5 \AA$ which could be related to the largest d-spacing observed $(50.3 \AA$ ). Finally, the d-spacing of $11.3 \AA$ could represent the hard domain between the urea motifs. These trends are observed in all MDI containing unsymmetrical bis-ureas demonstrating selfassembly of the bis-urea moieties. Lamellar structures are observed in HDI derived unsymmetrical bisureas, but associated with a smaller layer spacing. Peaks are noted between 31.7- 29.2 $\AA$, and 15.9-15.4 
$\AA$ for all samples which can be assigned to the first and second order reflections from a lamellar structure. WAXS analysis (Figure 7b) also exhibited d-spacings of $2.2 \AA$ and $1.7 \AA$ which are typical length scales found in hydrogen bonded ureas and urethanes,[36] providing further proof of the self-assembled array through hydrogen bonds. Furthermore, the $4.4 \AA$ WAXS peak can be assigned to intermolecular spacing in the supramolecular arrays.[37] The presence of higher order peaks at $2.2 \AA$ and $1.7 \AA$ may suggest local hexagonal ordering (peak positions in the ratio $1: 2: \sqrt{7}$ approximately), although the higher intensity of the $2.2 \AA$ peak is not consistent with the typical decrease in intensity with the reflection order observed for such a structure so these peaks may be due to other local packing features.
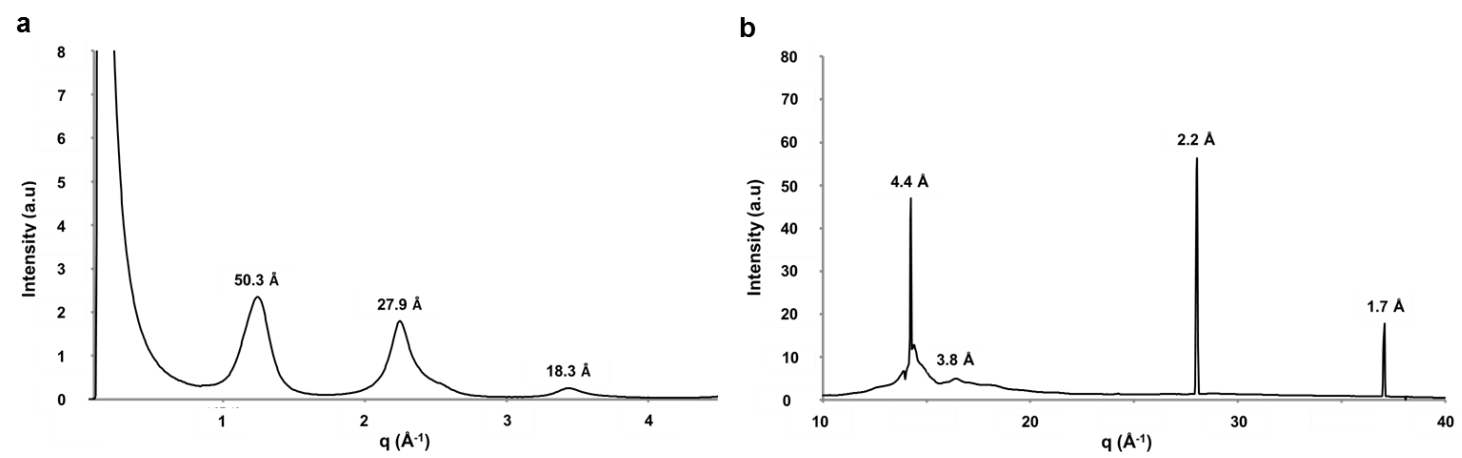

Figure 7: a) SAXS and b) WAXS data acquired for bis-urea 8 . The d-spaceings are shown above each peak.

Variable temperature SAXS analysis was also carried out on the unsymmetrical bis-ureas 8-15. The intensity of the peaks, corresponding to aggregation and hydrogen bonding, were seen to diminish as the temperature was elevated from $0{ }^{\circ} \mathrm{C}$ to $200{ }^{\circ} \mathrm{C}$ before cooling to $0{ }^{\circ} \mathrm{C}$ once again in $10{ }^{\circ} \mathrm{C}$ increments. A general trend of shifts to larger d-spacings was also observed as the temperature approached the melting point of the unsymmetrical bis-ureas. Indeed between $110-130{ }^{\circ} \mathrm{C}$, a new morphology was observed in both 4,4'-MDI and HDI derived bis-ureas as evidenced by large shifts in the d-spacing of the supramolecular array (Figures S17 and S18) which correlates with the thermal analysis data (Figure 5 and Table S2). Upon surpassing this temperature range, all higher-order structure diminished as the supramolecular array disassembles. This change in morphology closely reflects the melts observed by DSC where a common isomorph is seen to melt in this temperature range. Upon cooling to room temperature, the supramolecular arrays are seen to reform, again evidenced by the appearance of peaks corresponding to the different morphologies present in the array.

${ }^{1} \mathrm{H}$ NMR spectroscopy experiments were designed to compare the two differing structures reported herein, and probe this hypothesis. The unsymmetrical bis-ureas synthesised 8-15 all showed good solubility in DMSO, allowing for characterisation by ${ }^{1} \mathrm{H}$ and ${ }^{13} \mathrm{C}$ NMR spectroscopy. However, to calculate association constants, a solvent which does not disrupt hydrogen bonding within the supramolecular array, for which DMSO is known to do so though non-covalent interactions with the hydrogen bonding motifs [14] was required. Thus solubility in chloroform was desirable. Owing to the 
poor solubility of the unsymmetrical bis-ureas in $\mathrm{CDCl}_{3}$, symmetrical bis-ureas $\mathbf{1 6}$ and $\mathbf{1 7}$ were synthesised (Figure S21 and S22) from their respective diisocyanates 1 or $\mathbf{2}$ and 2-ethyl-1-hexylamine 3 (Scheme 2) to assess the rôle of the bridging unit between the ureas in self-assembly of the supramolecular array.

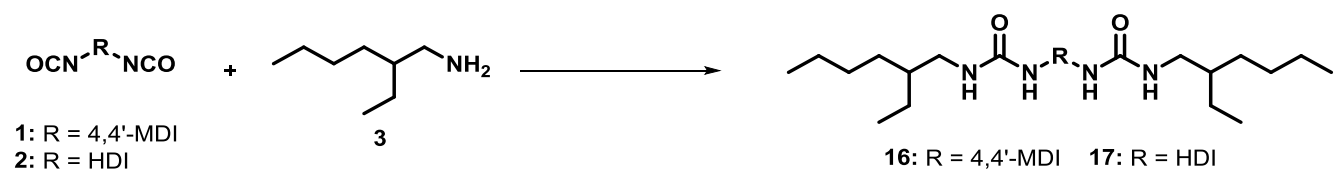

Scheme 2: Synthetic route to symmetrical bis-ureas 16 and 17 with desirable solubility characteristics in chloroform for association constant studies.

Solutions of the bis-ureas 16 (Figure 8a) or $\mathbf{1 7}$ (Figure 8b) were dissolved in deuterated chloroform at known concentrations between $0.001 \mathrm{M}$ and $0.190 \mathrm{M}$ and the chemical shift of two probe resonances (-NHC(O)NH-) recorded in a procedure previously described by Meijer and co-workers.[38]
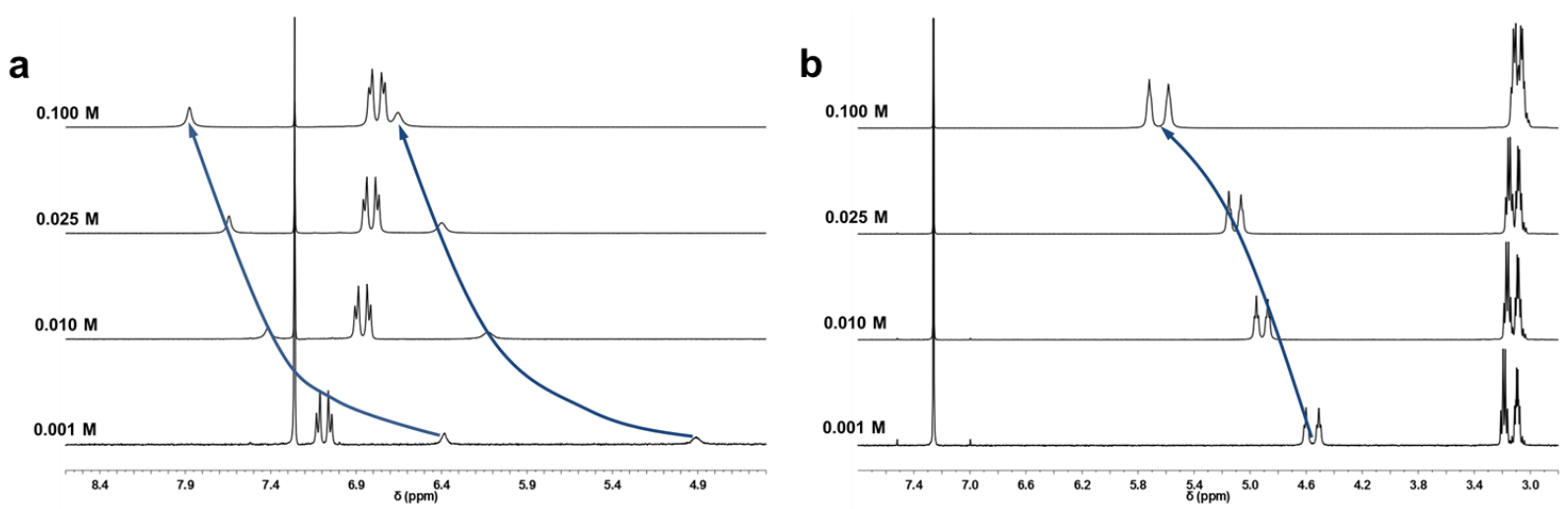

Figure 8: ${ }^{1} \mathrm{H}$ NMR Spectra of a) $\mathbf{1 6}$ and b) 17 at varying concentrations, showing the downfield shift $\mathrm{N} H$ urea resonances used to determine the association constants.

DynaFit[30] software was then utilised to determine[39] the association constant, which revealed that the central bridging unit between the hydrogen bonding components (i.e. aromatic 4,4'-MDI or aliphatic HDI cores) does indeed impact upon the association constant significantly. When an aromatic bridging unit was present $(\mathbf{1 6})$, association constants $\left(K_{\mathrm{a}}\right)$ of $182 \mathrm{M}^{-1}$ were observed. In contrast, when an aliphatic bridge was incorporated (17), the association constant was observed to drop to $K_{\mathrm{a}}=26 \mathrm{M}^{-1}$. These association constants reveal that the capability for $\pi-\pi$ interactions in $4,4^{\prime}$-MDI derived molecule 16 increases the binding affinity when compared to the aliphatic HDI derived analogue $\mathbf{1 7}$ as assist in the formation of supramolecular arrays which are more robust with respect to temperature, as observed by DSC and X-ray analysis. The increase in association constant when compared to those reported by Woodward et al[34] further reveal the importance of molecular design in tuning the physical properties of urea containing materials. 
Previous binding studies by Woodward et al.[14,34] on structurally related bis-urethane model compounds featuring a morpholine and 2-ethyl-1-hexyl end group, demonstrated a $K_{\mathrm{a}}$ of $15 \mathrm{M}^{-1}$ as derived by ${ }^{1} \mathrm{H}$ NMR spectroscopy. It was shown that the central hydrogen bonding motifs, i.e. carbamate (urethane) or carbamide (urea), play a far greater role in the self-assembly of such small molecules than the contributions of end-groups. Indeed, ureas revealed association constants which were approximately an order of magnitude greater than the urethane analogues. To further increase the association constant, we propose that an aromatic bridging unit between the hydrogen bonding motif, namely 4,4'-MDI 1 would further enhance the strength of these supramolecular interactions.

\section{Conclusion}

We have demonstrated a facile experimental procedure to produce unsymmetrical bis-ureas which can be harnessed as building blocks for incorporating hydrogen bonding motifs into higher order structures. Evidence of self-assembly was confirmed from the presence of dimers observed by mass spectroscopy, and infrared spectroscopy in the bulk. A structure-property relationship study has revealed that the thermal properties of the unsymmetrical bis-urea can be selectively tuned through varying the spacer length between the terminal hydroxyl group and adjacent urea. Indeed, understanding of these parameters enables control of the reversible supramolecular arrays formed between the self-complementary moieties. The ambient temperature SAXS data show the ability for the mono hydroxy-alkyl derivatives to arrange into highly ordered structures, particularly in the case of the MDI analogues; it is therefore proposed that a similar mechanism related to the aromaticity is responsible for both the increased association constant observed in the MDI model compound and the ordered structures evident from the SAXS studies. Indeed, the introduction of unsymmetrical bis-ureas increases the complexity of the self-assembled arrays through formation of different hydrogen bonding interactions between the various motifs present. This data was confirmed by DSC experiments which revealed thermal reorganisation of the self-assembled arrays as a function of temperature into the different morphologies described by molecular modelling. Finally, the small molecules reported here exhibit greater than 15 fold increase in association constant compared to their bis-urethane analogues previously reported and have also established the importance of $\pi-\pi$ stacking in molecular recognition and therefore provide an optimum route to forming supramolecular architectures.

\section{Acknowledgements}

Henkel Adhesives Ltd. are gratefully acknowledged for financial support for the $\mathrm{PhD}$ studentship for KM. We acknowledge the European Synchrotron Radiation Facility for provision of synchrotron radiation facilities and we would like to thank Dr Giuseppe Portale for assistance in using beamline BM26B. We thank the Chemical Analysis Facility of the University of Reading for access to thermal and spectroscopic analytical equipment. 


\section{References}

[1] M.A. Cohen Stuart, Colloid Polym. Sci. 286 (2008) 855-864.

[2] L. Fabbrizzi, A. Poggi, Chem. Soc. Rev. 24 (1995) 197-202.

[3] N.M. Sangeetha, U. Maitra, Chem. Soc. Rev. 34 (2005) 821-836.

[4] J.-M. Lehn, Chem. Soc. Rev. 36 (2007) 151-160.

[5] L.J. Prins, D.N. Reinhoudt, P. Timmerman, Angew. Chem., Int. Ed. 40 (2001) 23822426.

[6] F.J.M. Hoeben, P. Jonkheijm, E.W. Meijer, A.P.H.J. Schenning, Chem. Rev. 105 (2005) 1491-546.

[7] L. Brunsveld, B.J. Folmer, E.W. Meijer, R.P. Sijbesma, Chem. Rev. 101 (2001) 40714098.

[8] R.F.M. Lange, M. Van Gurp, E.W. Meijer, J. Polym. Sci. Part A Polym. Chem. 37 (1999) 3657-3670.

[9] L.R. Hart, J.L. Harries, B.W. Greenland, H.M. Colquhoun, W. Hayes, Polym. Chem. 4 (2013) 4860-4870.

[10] C.B. Aakeroy, A.M. Beatty, B.A. Helfrich, Angew. Chemie - Int. Ed. 40 (2001) 32403242.

[11] R.P. Sijbesma, F.H. Beijer, L. Brunsveld, B.J.B. Folmer, J.H.K. Ky Hirschberg, R.F.M. Lange, J.K.L. Lowe, E.W. Meijer, Science. 278 (1997) 1601-1604.

[12] H. Heterocomplex, T. Park, S.C. Zimmerman, (2006) 11582-11590.

[13] S. Bhattacharya, S.N.G. Acharya, Chem. Mater. 11 (1999) 3121-3132.

[14] P. Woodward, A. Clarke, B.W. Greenland, D. Hermida Merino, L. Yates, A.T. Slark, J.F. Miravet, W. Hayes, Soft Matter. 5 (2009) 2000-2010.

[15] B. Isare, G. Pembouong, F. Boué, L. Bouteiller, Langmuir. 28 (2012) 7535-7541.

[16] F. Schoonbeek, van Esch Jan H., R. Hulst, R.M. Kellogg, B.L. Feringa, Chem. - A Eur. J. 6 (2000) 2633-2643.

[17] F. Rodriguez-Llansola, D. Hermida-Merino, B. Nieto-Ortega, F.J. Ramirez, J.T.L. Navarrete, J. Casado, I.W. Hamley, B. Escuder, W. Hayes, J.F. Miravet, Chem. - A Eur. J. 18 (2012) 14725-14731.

[18] O.J. Dautel, M. Robitzer, J.-P. Lere-Porte, F. Serein-Spirau, J.J.E. Moreau, J. Am. Chem. Soc. 128 (2006) 16213-16223.

[19] A. Lapprand, F. Boisson, F. Delolme, F. Méchin, J.-P. Pascault, Polym. Degrad. Stab. 90 (2005) 363-373.

[20] J.H. van Esch, B.L. Feringa, Angew. Chemie - Int. Ed. 39 (2000) 2263-2266.

[21] P. Terech, R.G. Weiss, Chem. Rev. 97 (1997) 3133-3159.

[22] P. Dastidar, Chem. Soc. Rev. 37 (2008) 2699-2715.

[23] M. Akazawa, K. Uchida, J.J.D. de Jong, J. Areephong, M. Stuart, G. Caroli, W.R. Browne, B.L. Feringa, Org. Biomol. Chem. 6 (2008) 1544-1547.

[24] D.M. Wood, B.W. Greenland, A.L. Acton, F. Rodríguez-Llansola, C.A. Murray, C.J. Cardin, J.F. Miravet, B. Escuder, I.W. Hamley, W. Hayes, Chem. - A Eur. J. 18 (2012) 2692-2699.

[25] N. Volz, J. Clayden, Angew. Chemie - Int. Ed. 50 (2011) 12148-12155.

[26] N.E. Botterhuis, S. Karthikeyan, A.J.. H. Spiering, R.P. Sijbesma, Macromolecules. 43 (2010) 745-751.

[27] R.A. Koevoets, R.M. Versteegen, H. Kooijman, A.L. Spek, R.P. Sijbesma, E.W. Meijer, J. Am. Chem. Soc. 127 (2005) 2999-3003.

[28] S. Boileau, L. Bouteiller, F. Laupretre, F. Lortie, New J. Chem. 24 (2000) 845-848.

[29] F. Lortie, S. Boileau, L. Bouteiller, C. Chassenieux, B. Deme, G. Ducouret, M. Jalabert, F. Laupretre, P. Terech, Langmuir. 18 (2002) 7218-7222.

[30] P. Kuzmic, Anal. Biochem. 237 (1996) 260-273.

[31] S.L. Mayo, B.D. Olafson, W.A. Goddard III, J. Phys. Chem. 94 (1990) 8897-8909.

[32] J. van Esch, F. Schoonbeek, M. de Loos, H. Kooijman, A.L. Spek, R.M. Kellogg, B.L. Feringa, Chem. - A Eur. J. 5 (1999) 937-950.

[33] P. Brocorens, M. Linares, C. Guyard-Duhayon, R. Guillot, B. Andrioletti, D. Suhr, B. 
Isare, R. Lazzaroni, L. Bouteiller, J. Phys. Chem. B. 117 (2013) 5379-5386.

[34] P.J. Woodward, D. Hermida Merino, B.W. Greenland, I.W. Hamley, Z. Light, A.T. Slark, W. Hayes, Macromolecules. 43 (2010) 2512-2517.

[35] H. Pan, Rapid Commun. Mass Spectrom. 22 (2008) 3555-3560.

[36] E. Yilgör, E. Burgaz, E. Yurtsever, I. Yilgör, Polymer (Guildf). 41 (2000) 849-857.

[37] I.W. Hamley, A. Dehsorkhi, V. Castelletto, J. Seitsonen, J. Ruokolainen, H. Iatrou, Soft Matter. 9 (2013) 4794-4801.

[38] F.H. Beijer, R.P. Sijbesma, H. Kooijman, A.L. Spek, E.W. Meijer, J. Am. Chem. Soc. 120 (1998) 6761-6769.

[39] B.W. Greenland, S. Burattini, W. Hayes, H.M. Colquhoun, Tetrahedron. 64 (2008) 8346-8354. 